\title{
Distancia entre el currículo de Educación Secundaria y Terciaria: desafío para la política pública e instituciones
}

\section{Distance between the curriculum of secondary and higher education: a challenge for public policy and institutions}

Gonzalo Fonseca Grandón ${ }^{1}$

\begin{abstract}
Resumen: el problema central que se aborda es la existencia o no de continuidad entre el currículo de la educación secundaria y el nivel de exigencia de la educación terciaria o superior. Para estudiarlo, se presenta un estudio que contempló al análisis del Marco Curricular de la Enseñanza Media (2005) y un cuestionario aplicado a estudiantes de primer año de universidad. Los resultados arrojan que sí existe una continuidad curricular entre ambos niveles educacionales, aun cuando hay algunas dimensiones (como Habilidades del aprendizaje y sus subcategorías) que presentan una ruptura parcial. La conclusión apunta a la necesidad de articular más estrechamente ambos currículos, para evitar la reprobación y la deserción en los primeros años universitarios.
\end{abstract}

Palabras clave: currículo escolar, currículo universitario, dimensiones del aprendizaje.

\begin{abstract}
: the central problem faced is the existence of a continuity between the curriculum of secondary education and the level of requirements of the higher education. In order to study this issue, it is presented a study which involved the analysis of the Curriculum Framework of High School (2005) and a questionnaire made to students in their first year of university. Results showed that there is a curricular continuity between the two educational levels, although there are some dimensions (such as learning skills and their subcategories) having a partial rupture. The conclusion suggests a need of connecting both kind of curricula in order to avoid fail a course and leave early the university.
\end{abstract}

Keywords: secondary school curriculum, university curriculum, dimensions of learning.

\section{Antecedentes generales}

El debate sobre el tránsito de los estudiantes desde el nivel secundario al terciario, es una preocupación que se ha venido incrementando durante la última década en nuestro país y actualmente constituye un tema de gran interés para la comunidad académica. Una de las causas de dicha preocupación está dada por el aumento de la matrícula de estudiantes en Educación Superior. "En el caso de la matrícula de Pregrado, existe un incremento progresivo durante los dos periodos de estudio (1990-2000-2010). La década de 1990 inicia con 245.561 estudiantes matriculados en Pregrado, el año 2000 se alcanzan los 435.884 estudiantes, y concluye al final de la década (Año 2009) con 835.247 estudiantes. Lo

\footnotetext{
${ }^{1}$ Académico Facultad de Educación. Jefe Departamento de Currículum Dirección de Docencia Universidad Católica de la Santísima Concepción, Concepción, Chile. Correo electrónico: gfonseca@ ucsc.cl
} 
anterior equivale a un incremento de más de tres veces la cantidad de estudiantes durante estos 20 años",

Por ejemplo, el siguiente cuadro muestra la evolución de la matrícula de primer año de profesionales y licenciaturas por tipo de institución.

\section{Cuadro N¹: Evolución de la Matrícula de Primer Año de profesionales y licenciaturas por tipo de Institución en Chile}

\begin{tabular}{|c|c|c|c|c|c|c|c|}
\hline \multirow{2}{*}{ Tipo Institución } & \multicolumn{6}{|c|}{ Matrícula de Profesionales y/o Licenciatura } & \multirow{2}{*}{$\begin{array}{l}\text { Variación } \\
2005-2010\end{array}$} \\
\hline & 2005 & 2006 & 2007 & 2008 & 2009 & 2010 & \\
\hline UNIV & 106,352 & 117,524 & 118,605 & 116,957 & 130,244 & 140,321 & $32 \%$ \\
\hline IP & 29,672 & 32,053 & 35,973 & 37,694 & 39,011 & 47,434 & $60 \%$ \\
\hline \multicolumn{8}{|l|}{$\mathrm{CFT}$} \\
\hline Total & 136,024 & 149,577 & 154,578 & 154,651 & 169,255 & 187,755 & $38 \%$ \\
\hline
\end{tabular}

Fuente: Proceso de Matrícula 2010, Consejo Nacional de Educación.

En este contexto, la necesidad de dar cabida a un número cada vez mayor de estudiantes en el espacio universitario tradicional, es solo el aspecto más evidente de un problema mucho más profundo: como adaptar las instituciones tradicionales a un conjunto completamente nuevo de grupos sociales, funciones y demandas ${ }^{3}$.

Lo anterior, hace destacar los esfuerzos realizados por el gobierno chileno y las instituciones de Educación Superior al atender este nuevo escenario con estudiantes provenientes de los quintiles con menos recursos y con debilidades en sus competencias básicas para iniciar estudios universitarios. De allí que surgen interrogantes como ¿qué hacer desde las universidades para favorecer la inserción de los jóvenes provenientes de la educación media?

El panorama a nivel Latinoamericano no es tan diferente a lo que ocurre en Chile, pues, la expansión masiva en el acceso a la Educación Superior ha sido una característica de varias naciones del continente. La tabla A muestra la evolución de la matrícula universitaria desde el año 1970 en países de América Latina y el Caribe, en la que se puede observar que la matrícula universitaria se ha incrementado en algunos países en más de diez veces.

\footnotetext{
${ }^{2}$ ROLANDO, R., SALAMANCA, V. y ALIAGA, J. M. Evolución Matrícula Educación Superior de Chile Periodo 1990 - 2009. Sistema Nacional de Información de la Educación Superior (SIES), División de Educación Superior del MINEDUC. Junio 2010.

${ }^{3}$ ALTBACH, P. y MCGILL, P., (Ed). Educación Superior en el siglo XXI; Desafío Global y Respuesta Nacional. Buenos Aires: Ed. Biblio, 2000. P. 79.
} 


\section{Cuadro $N^{\circ}$ 2: Matrícula en Educación Superior por año}

\begin{tabular}{|c|c|c|c|c|c|c|c|c|c|}
\hline Pais & 1970 & 1980 & 1990 & 1995 & 1999 & 2000 & 2004 & 2005 & 2006 \\
\hline Argentina & $\begin{array}{c}274 \\
634,0\end{array}$ & $\begin{array}{c}491 \\
473,0 \\
\end{array}$ & $\ldots$ & $\ldots$ & $\begin{array}{l}1600 \\
882,0\end{array}$ & $\begin{array}{l}1766 \\
933,0\end{array}$ & 2116876,0 & 2082577,0 & $\ldots$ \\
\hline Bolivia & 35250,0 & $\ldots$ & $\ldots$ & $\ldots$ & $\begin{array}{c}252 \\
706,0\end{array}$ & 278763,0 & 346056,0 & $\ldots$ & $\ldots$ \\
\hline Brasil & $\begin{array}{c}430 \\
473,0\end{array}$ & $\begin{array}{l}1409 \\
243,0\end{array}$ & 1540080,0 & $\ldots$ & $\begin{array}{l}2456 \\
961,0\end{array}$ & $\begin{array}{l}2781 \\
328,0\end{array}$ & 4275027,0 & 4572297,0 & $\ldots$ \\
\hline Chile & 78430,0 & $\begin{array}{c}145 \\
497,0\end{array}$ & $\ldots$ & 342788,0 & $\begin{array}{c}450 \\
952,0 \\
\end{array}$ & 452177,0 & 580815,0 & 663694,0 & $\begin{array}{c}661 \\
142,0 \\
\end{array}$ \\
\hline Colombia & 85560,0 & $\begin{array}{c}271 \\
630,0 \\
\end{array}$ & 487448,0 & 588322,0 & $\begin{array}{c}877 \\
944,0 \\
\end{array}$ & 934085,0 & 1112574,0 & 1223594,0 & $\begin{array}{l}1314 \\
972,0 \\
\end{array}$ \\
\hline Costa Rica & 15473,0 & 55593,0 & 74681,0 & $\cdots$ & 58761,0 & 61654,0 & 108765,0 & 110717,0 & $\cdots$ \\
\hline Cuba & 26342,0 & $\begin{array}{c}151 \\
733,0 \\
\end{array}$ & 242434,0 & 122346,0 & $\begin{array}{c}153 \\
463,0 \\
\end{array}$ & 158674,0 & 396516,0 & 471858,0 & $\begin{array}{c}681 \\
629,0 \\
\end{array}$ \\
\hline Ecuador & 38692,0 & $\begin{array}{c}269 \\
775,0 \\
\end{array}$ & 206541,0 & $\ldots$ & $\ldots$ & $\ldots$ & $\ldots$ & $\ldots$ & $\ldots$ \\
\hline Guatemala & 15609,0 & 50890,0 & $\ldots$ & 80228,0 & $\ldots$ & $\ldots$ & $\ldots$ & $\ldots$ & $\begin{array}{c}112 \\
215,0\end{array}$ \\
\hline México & $\begin{array}{c}247 \\
637,0 \\
\end{array}$ & $\begin{array}{c}929 \\
865,0 \\
\end{array}$ & 1310835,0 & 1532846,0 & $\begin{array}{l}1837 \\
884,0\end{array}$ & $\begin{array}{l}1962 \\
763,0 \\
\end{array}$ & 2322781,0 & 2384858,0 & $\begin{array}{l}2446 \\
726,0 \\
\end{array}$ \\
\hline Panamá & 8947,0 & 40369,0 & 53235,0 & 76798,0 & $\begin{array}{c}108 \\
764,0\end{array}$ & 118502,0 & 128558,0 & 126242,0 & $\begin{array}{r}130 \\
838,0\end{array}$ \\
\hline Paraguay & 8172,0 & 26915,0 & 32884,0 & 40913,0 & 66065,0 & 83088,0 & 149120,0 & 156167,0 & $\ldots$ \\
\hline Perú & $\begin{array}{c}126 \\
234,0\end{array}$ & $\begin{array}{c}306 \\
353,0 \\
\end{array}$ & 678236,0 & 671802,0 & $\ldots$ & $\ldots$ & 896501,0 & 909315,0 & $\begin{array}{r}952 \\
437,0 \\
\end{array}$ \\
\hline R. Dominicana & 23546,0 & $\ldots$ & $\ldots$ & $\ldots$ & $\ldots$ & $\ldots$ & 293565,0 & $\ldots$ & $\ldots$ \\
\hline Uruguay & $\ldots$ & 36298,0 & 71612,0 & $\ldots$ & 91275,0 & 97641,0 & 103431,0 & 110684,0 & $\begin{array}{c}113 \\
368,0\end{array}$ \\
\hline Venezuela & $\begin{array}{c}100 \\
767,0\end{array}$ & $\begin{array}{c}307 \\
133,0 \\
\end{array}$ & 550030,0 & $\ldots$ & $\ldots$ & 668109,0 & 1049780,0 & $\cdots$ & $\begin{array}{l}1381 \\
126,0 \\
\end{array}$ \\
\hline
\end{tabular}

Fuente: CEPAL. Estadísticas de América Latina y el Caribe.

La temática de la retención de estudiantes muestra cuatro vertientes analíticas sobre las cuales es recomendable poner mayor atención, la primera es aquella de la cual tenemos mayores antecedentes en nuestro medio: (i) la relación entre las características previas del sujeto y su éxito en los primeros años en la universidad; (ii) identificar los aspectos a los cuales los estudiantes le atribuyen importancia antes de graduarse; (iii) la descripción de experiencias diseñadas para incrementar la retención (área sobre la cual se han centrado en la actualidad la mayor parte de los trabajos sobre el tema), y (iv) análisis de los resultados 
de procesos de innovación pedagógica y sus impactos en la retención (dimensión que está en pleno desarrollo) ${ }^{4}$.

\section{Objetivo del estudio}

Identificar las continuidades y discontinuidades entre el currículo de la enseñanza secundaria y el de enseñanza superior mediante un estudio de caso, proponiendo desafíos para la política pública e instituciones.

\section{Metodología}

La metodología utilizada en este estudio fue de carácter cualitativo-interpretativa. Se seleccionó una muestra intencionada que consideró doce proyectos de diversas carreras, de distintas áreas, pertenecientes a una Universidad. Se estudiaron agrupadamente todas aquellas dimensiones que las carreras evaluaban en sus estudiantes a su ingreso a la universidad, porque eran consideradas necesarias para iniciar sus estudios Además, se identificó aquellas dimensiones evaluadas institucionalmente por la universidad. A partir de las dimensiones identificadas y evaluadas tanto por las carreras como por la institución, se indagó y analizó el Marco Curricular de la Educación Media chilena, para determinar los aprendizajes explícitos y terminales declarados en cada una de las dimensiones identificadas.

El análisis fue realizado a partir de lo recolectado desde dos perspectivas, una de carácter prescriptivo, a partir de lo explicitado en el Marco Curricular de la Educación Media chilena y otra de carácter declarativo, a partir de la información recolectada luego de la aplicación de un cuestionario para conocer antecedentes de las dimensiones evaluadas a los estudiantes que ingresan a primer año, para posteriormente realizar la triangulación.

La recolección de la información consideró la aplicación de un cuestionario que fue parte de la ejecución de un Proyecto desarrollado con recursos del Fondo de Desarrollo Institucional del Ministerio de Educación de Chile en el marco del desarrollo del Proyecto: "El proceso de transición entre Educación Media y Superior", ejecutado en conjunto con las universidades pertenecientes al Grupo Operativo de CINDA $^{5}$ y, por otro lado, un análisis documental acabado del Marco Curricular de la Educación Media chilena6 (Actualización 2005), a cargo de una de las instituciones participantes en el Proyecto.

Para la contrastación de la información, se realizó un análisis de contenido a partir de las categorías identificadas previamente (quince dimensiones) y lo explicitado en el

\footnotetext{
4 “Análisis de los modelos explicativos de retención de estudiantes en la universidad: una visión desde la desigualdad social”. Estudios Pedagógicos. 2007, vol. XXXIII, núm. 1. P. 7-27.

${ }^{5}$ Centro Interuniversitario de Desarrollo, que en Chile agrupa a un conjunto de catorce Universidades pertenecientes al Consejo de Rectores de Universidades Chilenas para debatir en temas de actualidad en Educación Superior.
} 
Marco Curricular de la Educación Media chilena ${ }^{6}$ en relación con dichas categorías. Se presenta una síntesis esquemática del modelo metodológico utilizado en la muestra la figura 1.

\section{Estudio de Caso de}

1. Quince dimensiones evaluadas a estudiantes de $1^{\text {a }}$ año de universidad de 12 proyectos de carrera de una Universidad.

2. Contrastación de las quince dimensiones identificadas anteriormente con el Marco Curricular de la Educación Media chilena.

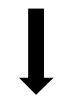

\section{Objetivo del Estudio}

Analizar las continuidades y discontinuidades entre el currículo de la Enseñanza Media y el de la Enseñanza Superior, mediante un estudio de caso proponiendo desafíos para la política pública e instituciones.

\section{Recolección de información:}

1. Aplicación de cuestionarios

2. Análisis del Marco Curricular de Triangulación de Datos la Educación Media chilena.

Contrastación de la información recolectada en los cuestionarios y la información del Marco Curricular de la Educación Media chilena.

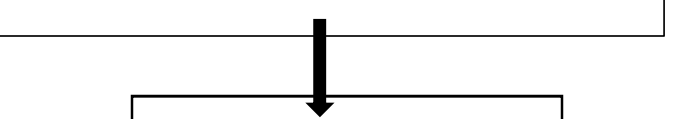

\section{Conclusiones}

\footnotetext{
${ }^{6}$ MINEDUC. Marco curricular de la Educación Media chilena. Objetivos fundamentales y contenidos
} mínimos obligatorios. Santiago de Chile, 2005. 
Cuadro $N^{\circ}$ 3: Dimensiones evaluadas por las carreras y/o instituciones y cobertura

\begin{tabular}{|l|l|l|}
\hline $\mathbf{N}^{\mathbf{o}}$ & DIMENSIÓN EVALUADA & Cobertura \\
\hline 1 & Estilos de aprendizaje & $85 \%$ \\
\hline 2 & Emprendimiento y liderazgo & $75 \%$ \\
\hline 3 & Características de la personalidad & $60 \%$ \\
\hline 4 & Comprensión lectora & $55 \%$ \\
\hline 5 & Comunicación/expresión escrita & $30 \%$ \\
\hline 6 & Habilidades sociales & $15 \%$ \\
\hline 7 & Expresión oral & $15 \%$ \\
\hline 8 & Razonamiento matemático & $15 \%$ \\
\hline 9 & Autoconcepto/autoimagen & $10 \%$ \\
\hline 10 & Autoestima & $10 \%$ \\
\hline 11 & Actitud hacia la responsabilidad social & $8 \%$ \\
\hline 12 & Estrategias de aprendizaje & $8 \%$ \\
\hline 13 & Estrategias metacognitivas & $>5 \%$ \\
\hline 14 & QPT INGLÉS & $>5 \%$ \\
\hline 15 & TIC & $>5 \%$ \\
\hline
\end{tabular}

Fuente: Elaboración a partir de análisis de cuestionario aplicado

\section{Resultados y análisis}

El análisis se desarrolló principalmente sobre la base de los objetivos fundamentales (con énfasis en los terminales) y contenidos mínimos obligatorios. A continuación, se presenta la contrastación realizada entre el marco curricular de la Educación Media chilena y las quince dimensiones identificadas anteriormente. Para el análisis comparativo entre las dimensiones indagadas en uno y otro nivel, se asimiló el concepto de "puente" o "sinergia", como un término que representa las variables en que debiera existir un tránsito expedito desde la Educación Media a la Educación Superior, y el concepto de "quiebre", para determinar en qué casos existe una ruptura, parcial o total, entre las dimensiones analizadas en ambos niveles educativos.

Para el cruce de información proveniente de ambas fuentes, se confeccionó una matriz que contrasta las quince dimensiones identificadas en el currículum de primer año de la Universidad con lo establecido en el Marco Curricular de la Educación Media chilena en torno a esas dimensiones, de modo determinar la distancia existente entre ambos niveles educativos para cada dimensión. Inmediatamente, se formula una breve reflexión que caracteriza la relación entre el currículum secundario y terciario para cada variable. Cada una de las quince dimensiones fue clasificada dentro de "categorías matrices" para simplificar la información y facilitar el análisis. 
Cuadro $\mathbf{N}^{\circ}$ 4: Matriz de categorías en donde existen quiebres o rupturas parciales entre el currículum de educación media y superior

CATEGORÍA MATRIZ: Habilidades del aprendizaje. (dimensiones incluidas: estilos de aprendizaje, estrategias de aprendizaje y estrategias metacognitivas).

\section{REFLEXIÓN}

Parece que no es coincidencia que las tres dimensiones en donde se encontraron rupturas parciales entre el currículum de la Educación Media y el de Educación Superior están vinculadas al aprendizaje de los estudiantes. El marco curricular, si bien explicita cuáles son las metas que los alumnos deben lograr, sólo alude implícitamente a que cada estudiante tiene características propias que lo diferencian de los demás, tanto en el estilo predominante de aprendizaje como en el conjunto de estrategias que utiliza un joven para aprender. No especifica los tipos de alumnos, según sus rasgos de orden psicológico, social, etc., y los estilos que a cada uno de estos tipos pudiera ser eficaz para un real aprendizaje, el cual pueda ser considerado por los establecimientos educacionales y por los docentes. Sólo deja entrever implícitamente un "téngase presente" respecto a la existencia de alumnos con distintas características y motivaciones. En consecuencia, las estrategias metacognitivas tampoco son favorecidas en la Enseñanza Media. Como antecedente, en la universidad se han establecido diferencias importantes de rendimiento asociadas a las estrategias metacognitivas en asignaturas de matemática y química. La Educación Secundaria no formula metas concretas para favorecer el trabajo de dichas dimensiones. Por lo tanto, se considera que existe un "quiebre" parcial entre el currículum de la Educación Media y Educación Superior para el caso de dimensiones que en la actualidad son necesarias para alcanzar aprendizajes con sentido.

Fuente: Elaboración para análisis de contenido 
Cuadro $\mathrm{N}^{\circ}$ 5: Matriz de categorías en donde existen puentes o sinergias parciales entre el currículum de educación media y el superior

CATEGORÍA MATRIZ: Habilidades de Inserción. (Dimensiones incluidas: emprendimiento y liderazgo, habilidades sociales y actitud hacia la responsabilidad social).

\section{REFLEXIÓN}

Emprendimiento, liderazgo, habilidades sociales y la actitud hacia la responsabilidad social, constituyen lo que para este estudio se denominan las habilidades de inserción. Son dimensiones presentes explícitamente en el Marco Curricular de la Educación Media y declaradas importantes por la institución. El desarrollo de dichas habilidades debería permitir a los estudiantes insertarse expeditamente en la vida universitaria, tomar decisiones progresivas en cuanto a su complejidad en el ámbito universitario, desenvolverse en situaciones académicas frecuentemente e insertarse hábilmente en actividades curriculares prácticas en diversas instituciones vinculadas al campo profesional.

CATEGORÍA MATRIZ: Habilidades comunicativas. (Dimensiones incluidas: comprensión lectora, comunicación: expresión escrita y expresión oral).

\section{REFLEXIÓN}

En rigor, la lectura y en consecuencia la comprensión lectora, la expresión escrita y la expresión oral, constituyen lo que se denomina habilidades comunicativas y son actividades habituales y transversales en el currículum la Universidad, al igual que la transversalidad manifestada en la declaración del Marco Curricular de la Educación Media. La expresión de ideas en un discurso frente a otros en el ámbito académico y/o personal y social de manera hablada, las lecturas bibliográficas, la presentación de trabajos escritos, entre otros, son actividades presentes en el currículum desde primer año en la Universidad y constituyen capacidades necesarias para favorecer un buen desempeño académico en varias actividades curriculares. Por lo tanto, existe una plena articulación entre el currículum declarado por ambos niveles educativos.

CATEGORÍA MATRIZ: Características de la personalidad. (Dimensiones incluidas: características de la personalidad, autoconcepto, autoimagen y autoestima).

\section{REFLEXIÓN}


Se ha utilizado la categoría características de la personalidad, para referirse al autoconcepto, autoimagen, autoestima y aquellas otras dimensiones propias de la personalidad que favorecen el tránsito entre un nivel y otro. El Marco Curricular presenta al estudiante de la Educación Secundaria como un joven respetuoso, crítico, perseverante, sociable, dinámico, seguro de sí mismo, capaz de adaptarse a distintas circunstancias para llevar adelante sus proyectos y desenvolverse adecuadamente en su futuro académico o laboral. El desarrollo de estos aprendizajes, permitiría a los estudiantes, afrontar diversas situaciones que surgen en su formación universitaria, en el ámbito familiar, y otros entornos relacionales. Constituyen cualidades presentes en varios perfiles de ingreso a la Universidad. Para el caso del autoconcepto o autoimagen, son principalmente los Objetivos Fundamentales Transversales los que determinan la presencia de ambas dimensiones en lo relacionado con el crecimiento y la autoafirmación personal. Para ello, la Educación Media debe destacar las experiencias formativas, incentivar los rasgos y características que formen parte de su identidad personal y elaborar estrategias para reforzarlas. Se debe lograr que sean conscientes de su rol dentro de la sociedad en la que se desenvuelven, que desarrollen su sentido de pertenencia tanto a la sociedad en su conjunto, como a diversos grupos en que participen. Esto le ayuda a establecer y mejorar sus conductas sociales, las relaciones con sus pares y la ayuda hacia los demás, lo que provoca que se sienta un hombre útil e importante; sociable y solidario; afectivo y aceptado. Es importante también para este Marco, favorecer el autoconocimiento, que le permita aceptarse tal como es y consciente de sus fortalezas y debilidades. Asimismo, el desarrollo de la afectividad y el equilibrio emocional, también se logra, profundizando en conceptos trascendentes como el amor y la amistad.

CATEGORÍA MATRIZ: Razonamiento matemático. (Dimensión incluida: razonamiento matemático).

\section{REFLEXIÓN}

El Marco Curricular, en efecto, propone y privilegia el desarrollo del razonamiento matemático, por sobre la aplicación de fórmulas sin un sentido lógico. Por otro lado, la Universidad evalúa el razonamiento lógico matemático y el conocimiento mínimo que deben tener los estudiantes de primer año principalmente en carreras de Ingeniería en materias de álgebra y cálculo. Si, a lo anterior, agregamos que la PSU también evalúa razonamiento matemático, estamos en presencia de una "triada" alineada.

CATEGORÍA MATRIZ: Otras categorías. (Dimensiones incluidas: inglés y TIC).

\section{REFLEXIÓN}

Para el caso de la Universidad, el inglés toma forma de cursos transversales en más del $90 \%$ de las carreras desde primer año, el plan de estudios de la Educación Media contempla el subsector de idioma extranjero, el que en amplia mayoría lo constituye el inglés. Para la dimensión TIC, existe articulación entre el currículum de educación media y superior. En ambos niveles educativos constituye una competencia transversal. Para la Educación Media, existen los objetivos fundamentales transversales de la informática. 


\section{Conclusiones}

En relación al tema central de este estudio, se concluye que existe articulación entre el currículum explícito declarado para la Educación Media chilena y el currículum de la universidad. Aun cuando el sentido de este análisis no es la generalización de los resultados, responsablemente se puede prever que un estudiante que egresa de la Educación Media chilena, debería transitar de manera expedita y natural hacia la Educación Superior, pues, para el caso de la institución estudiada, las coincidencias o articulaciones entre las quince variables identificadas, en general dan cuenta de un "camino despejado", aun cuando se producen algunos quiebres parciales en tres dimensiones pero que de ningún modo constituyen rupturas totales entre el currículum de un nivel y otro, pero que son importantes de profundizar, pues, constituyen variables vinculadas al aprendizaje.

Uno de los hallazgos en relación a los resultados obtenidos en la evaluación de las dimensiones identificadas en este estudio, indica que aún se requiere fortalecer las decisiones que se toman a partir de dichos resultados, es decir, está instalado el concepto de "función administrativa" de la evaluación. En general, las acciones implementadas a partir de los resultados, implican una acción reactiva de la evaluación, lo que se ve incrementado por el momento en que se aplican las evaluaciones, ya que todos los instrumentos son aplicados durante el primer semestre o al ingreso de los jóvenes a la Universidad. En consecuencia, las decisiones se toman a destiempo. Por otro lado, se observa que las acciones implementadas son aún aisladas y hay ausencia de seguimiento sistemático. En consecuencia, se percibe, en general, que el fracaso del estudiante en la Educación Media y Superior, es responsabilidad exclusiva de él, eximiendo de responsabilidad a la institución y/o a los profesores o académicos.

A modo de reflexión, cabe preguntarse: si existe articulación entre el currículum explícito de ambos niveles, ¿por qué existen altas tasas de reprobación, de deserción, bajas tasas de titulación oportuna, etc.? ¿La Educación Media chilena está logrando las metas propuestas en todos sus estudiantes? Parece que estas inquietudes son las que dejan abierto este estudio a futuras investigaciones, no obstante de él se extraen algunas recomendaciones generales, a corto y mediano plazo, para favorecer el tránsito desde un nivel a otro.

\section{Recomendaciones}

\section{A nivel institucional}

Es muy recomendable que la Universidad inicie acciones de articulación desde la Educación Media, previo al ingreso de los estudiantes a la institución y viceversa, favoreciendo la vinculación con la Educación Superior en un momento oportuno. Al respecto, lo anterior genera espacios de orientación vocacional a los jóvenes con un 
conocimiento adecuado de las carreras $\mathrm{y}$, por otra parte, permite a las universidades presentar su oferta educativa, entre otros.

Otra recomendación, tiene que ver con la necesidad de formular perfiles de ingreso a la universidad en términos de aprendizaje que sean esenciales para iniciar estudios en la Institución. Estos debieran orientar, efectivamente, la toma de decisiones para una conveniente planificación del primer año de universidad con espacios para la nivelación. En este mismo contexto, es necesario superar el concepto de "función administrativa" de la evaluación, de modo que se evalúe dimensiones intencionadamente y se tomen decisiones coherentes con los resultados de la evaluación.

Queda la sensación que el marco curricular de la Educación Media chilena constituye un documento amplio, general y un tanto ambicioso, en donde la transversalidad de ciertos objetivos hace asumir la responsabilidad de todos y nadie al mismo tiempo. Por ejemplo, ambos niveles educativos otorgan un espacio importante a las competencias transversales y/o genéricas, pero dicha transversalidad requiere que la instituciones y los profesores asuman la responsabilidad de desarrollarlos.

Aun cuando existe articulación curricular entre ambos niveles, todo hace prever que las metas propuestas para la Enseñanza Media no están siendo alcanzadas por los estudiantes. Existe allí una ruptura que puede ser por diferencias en los énfasis, las formas de generar aprendizajes, el tipo de aprendizaje logrado, entre otras. Por otro lado, el ritmo de estudio y trabajo académico es diferente. Sin embargo lo anterior, parece no ser considerado de manera óptima en la planificación curricular de la universidad. Las universidades, entonces, pueden impulsar intervenciones posteriores al ingreso, tales como unidades de apoyo al aprendizaje, seguimiento a estudiantes de mayor vulnerabilidad, levantamiento de indicadores $y$ bases de datos para favorecer un seguimiento individualizado, entre otros.

Se considera que el apoyo desde la universidad al sistema escolar puede contribuir al propósito que todos los jóvenes que ingresan al sistema puedan completar su Educación Media con las competencias necesarias para continuar estudios superiores. Por lo pronto, ofrecer programas de inserción a la universidad para los jóvenes provenientes de liceos municipales, fortalecer la orientación y consejería vocacional, favorecer el seguimiento de estudiantes de mayor vulnerabilidad, constituyen algunas acciones inmediatas que pueden ser implementadas en ambos niveles.

\section{A nivel de política pública}

En primer lugar, se plantea como recomendación una intervención sensible y profunda a nivel curricular en la Educación Media, tendiente a generar grandes áreas de conocimiento que permitan dar una mayor integración a los conocimientos de ese nivel, reduciendo la cantidad de sectores y subsectores de aprendizaje, evitando así la parcelación de los contenidos, pero por sobre todo otorgando espacios para la creación de un proyecto de vida profesional y de educación para la vida a los jóvenes de la Educación Media. Lo anterior, implicaría favorecer el trabajo profundo en áreas como: lenguaje, ciencias, 
matemática, idioma extranjero y proyecto de vida, entre las de base, otorgando amplios espacios para la formación ética y ciudadana.

Por otro lado, la Prueba de Selección Universitaria, constituye mayoritariamente la vía de ingreso a la universidad, principalmente a las del $\mathrm{CRUCH}$, por lo que incentivar a las universidades a diversificar las vías de admisión, podría constituir un espacio para otorgar mayor flexibilidad al sistema de ingreso a la universidad y generar mayor equidad, favoreciendo la participación de estudiantes pertenecientes a los quintiles más pobres.

Por último, proponer un modelo de articulación entre educación media y superior, que implique la creación de un organismo universitario o gubernamental, que haga más fluido el proceso de transición entre ambos niveles, considerando instancias compartidas de coordinación, sobre la base de acciones de discriminación positiva que favorezcan la equidad en el acceso y apoyo temprano, puede fortalecer y transparentar esta transición.

\section{Referencias bibliográficas}

ALTBACH, P. y MCGILL, P. (Eds.). Educación Superior en el siglo XXI; Desafío Global y Respuesta Nacional. Buenos Aires: Biblio, 2000.

COMISIÓN ECONÓMICA PARA AMÉRICA LATINA Y EL CARIBE, CEPAL. (Comp.). Estadísticas de América Latina y el Caribe. CEPAL, 2008. Disponible en web: www.cepal.org [consultado el 15 de marzo de 2012].

DONOSO, S. y SCHIEFELBEIN, E. "Análisis de los modelos explicativos de retención de estudiantes en la universidad: una visión desde la desigualdad social". En: Estudios Pedagógicos. 2007, vol. XXXIII, núm. 1. Valdivia.

MINEDUC. Marco Curricular de la Educación Media chilena. Objetivos fundamentalesy contenidos mínimos obligatorios. Santiago de Chile, 2005.

ROLANDO, R., SALAMANCA, V. y ALIAGA, J. M. Evolución Matrícula Educación Superior de Chile Periodo 1990 - 2009. Sistema Nacional de Información de la Educación Superior (SIES), División de Educación Superior del MINEDUC. Junio 2010.

División de Educación Superior del MINEDUC. Junio 2010.UNIVERSIDAD CATÓLICA DE LA SANTÍSIMA CONCEPCIÓN. Marco Curricular de la UCSC. Concepción, Chile, 2006. 